\title{
Ripplocations in van der Waals Layers
}

\author{
Akihiro Kushima, ${ }^{\dagger, \ddagger}$ Xiaofeng Qian, ${ }^{\dagger, \ddagger}$ Peng Zhao, ${ }^{\S}$ Sulin Zhang, ${ }^{*},+, \S$ and Ju Li ${ }^{*}, \dagger, \ddagger$ \\ ${ }^{\dagger}$ Department of Nuclear Science and Engineering and Department of Materials Science and Engineering, Massachusetts Institute of \\ Technology, 77 Massachusetts Avenue, Cambridge, Massachusetts 02139, United States \\ ${ }^{\S}$ Department of Engineering Science and Mechanics, Pennsylvania State University, University Park, Pennsylvania 16802, United \\ States
}

Supporting Information

ABSTRACT: Dislocations are topological line defects in three-dimensional crystals. Same-sign dislocations repel according to Frank's rule $\left|\mathbf{b}_{1}+\mathbf{b}_{2}\right|^{2}>$ $\left|\mathbf{b}_{1}\right|^{2}+\left|\mathbf{b}_{2}\right|^{2}$. This rule is broken for dislocations in van der Waals (vdW) layers, which possess crystallographic Burgers vector as ordinary dislocations but feature "surface ripples" due to the ease of bending and weak vdW adhesion of the atomic layers. We term these line defects "ripplocations" in accordance to their dual "surface ripple" and "crystallographic dislocation" characters. Unlike conventional ripples on noncrystalline (vacuum,

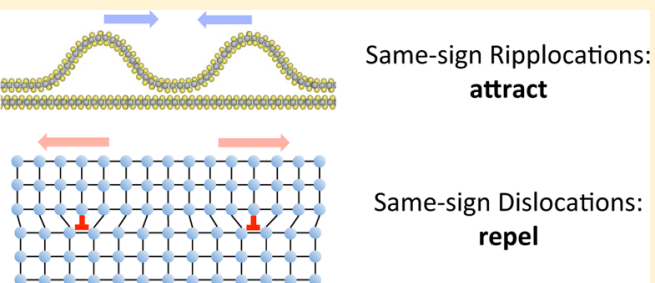
amorphous, or fluid) substrates, ripplocations tend to be very straight, narrow, and crystallographically oriented. The selfenergy of surface ripplocations scales sublinearly with $|\mathbf{b}|$, indicating that same-sign ripplocations attract and tend to merge, opposite to conventional dislocations. Using in situ transmission electron microscopy, we directly observed ripplocation generation and motion when few-layer $\mathrm{MoS}_{2}$ films were lithiated or mechanically processed. Being a new subclass of elementary defects, ripplocations are expected to be important in the processing and defect engineering of vdW layers.

KEYWORDS: 2D layered crystals, van der Waals homostructures, $\mathrm{MoS}_{2}$, ripple, dislocation

$\mathrm{S}^{\mathrm{h}}$ harp and localized folds ${ }^{1,2}$ have been observed in twodimensional (2D) layered crystals, ${ }^{3-9}$ when a top atomic layer slips against the bottom layer by an in-plane Bravais vector b. At the border between the slipped and unslipped areas, an excessive line of atoms can be regarded as being locally inserted into one layer relative to the other (as edge dislocation), creating a local line of ripple in van der Waals (vdW) homostructures. Such line defects in thin-layered crystals are topologically identical to but energetically distinct from conventional dislocations in bulk crystals due to the thinness of the vdW structures, the simplest of which is a bilayer, and they are hereafter termed ripplocations. In this study, ripplocations in thin layered $\mathrm{MoS}_{2}$ crystals and their motion under electrochemical lithiation and mechanical perturbation are atomically resolved with high-resolution transmission microscopy (HRTEM). Density functional theory (DFT) calculations reveal that ripplocations are highly localized with a core width of only a few nanometers. Same-signed ripplocations attract at short distances, conforming to the general "curve-to-attract" rule ${ }^{10}$ in membrane mechanics, and are weakly repulsive to each other at long distances. The energetics suggests that ripplocations tend to merge into larger, immobile folds. The presence of ripplocations is found to markedly alter the electronic transport properties of the layered crystals.

$\mathrm{MoS}_{2}$ thin films prepared by the scotch tape exfoliation technique ${ }^{11}$ were used in this study. A TEM image of the freestanding $\mathrm{MoS}_{2}$ thin film attached on an $\mathrm{Al}$ rod using conducting epoxy is shown in Figure 1a. Sharp lines aligned with well-defined crystallographic directions were observed in the thin film. A higher magnification image of the marked area in Figure 1a is shown in Figure $1 \mathrm{~b}$. The electron diffraction pattern (EDP) shown in the inset indicates that the sharp lines in the selected area are perpendicular to the $[10 \overline{1} 0]$ direction. Sharp lines in other crystallographic directions were also observed (see Supporting Information Figure S2). These sharp lines were commonly seen on many of the scotch tape exfoliated $\mathrm{MoS}_{2}$ film samples. According to the HRTEM analysis presented below, they were confirmed to be ripplocations formed on the surface layers with $\mathbf{b}=[10 \overline{1} 0]$, as schematically illustrated in Figure 1c.

Figure 1d displays an HRTEM image of a typical ripplocation observed in the films. Across the ripplocation the film maintained its perfect crystalline structure without exhibiting any broken bonds. A slight change in the achromatic contrast of width $\lambda \sim 5 \mathrm{~nm}$ was observed at the ripplocations, which was identified as the characteristic core width of a unit ripplocation (with unit Burgers vector $\mathbf{b}_{0}$ ) on the $\mathrm{MoS}_{2}$ thin film. The nanometer sharpness of the lines is distinct from the long-range bending morphology of an atomic film freely suspended or on an amorphous (noncrystalline) substrate. High-resolution cross-section image of a multilayered $\mathrm{MoS}_{2}$ (Figure 1e) shows ripplocations inside the crystalline film, evidencing the existence of membrane bending and extra lines of atoms in one $\mathrm{MoS}_{2}$ layer.

Received: November 24, 2014

Revised: December 15, 2014 

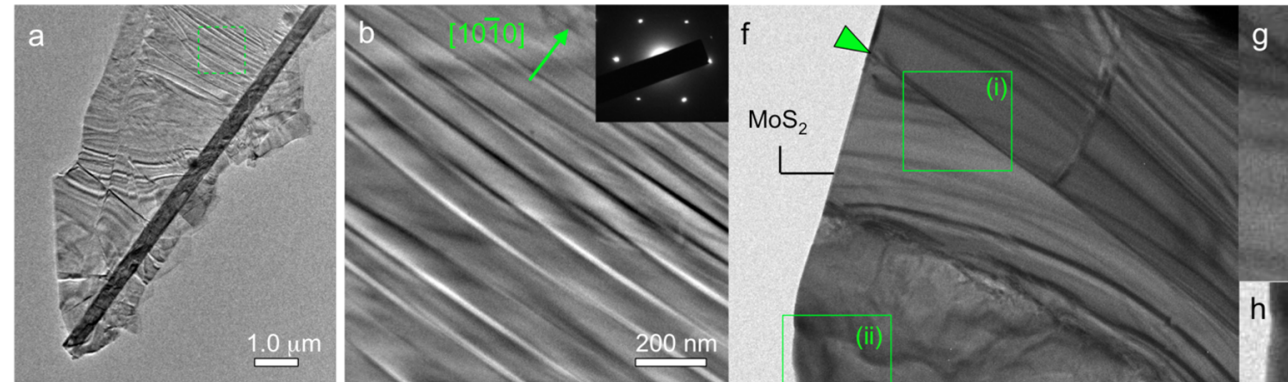

C
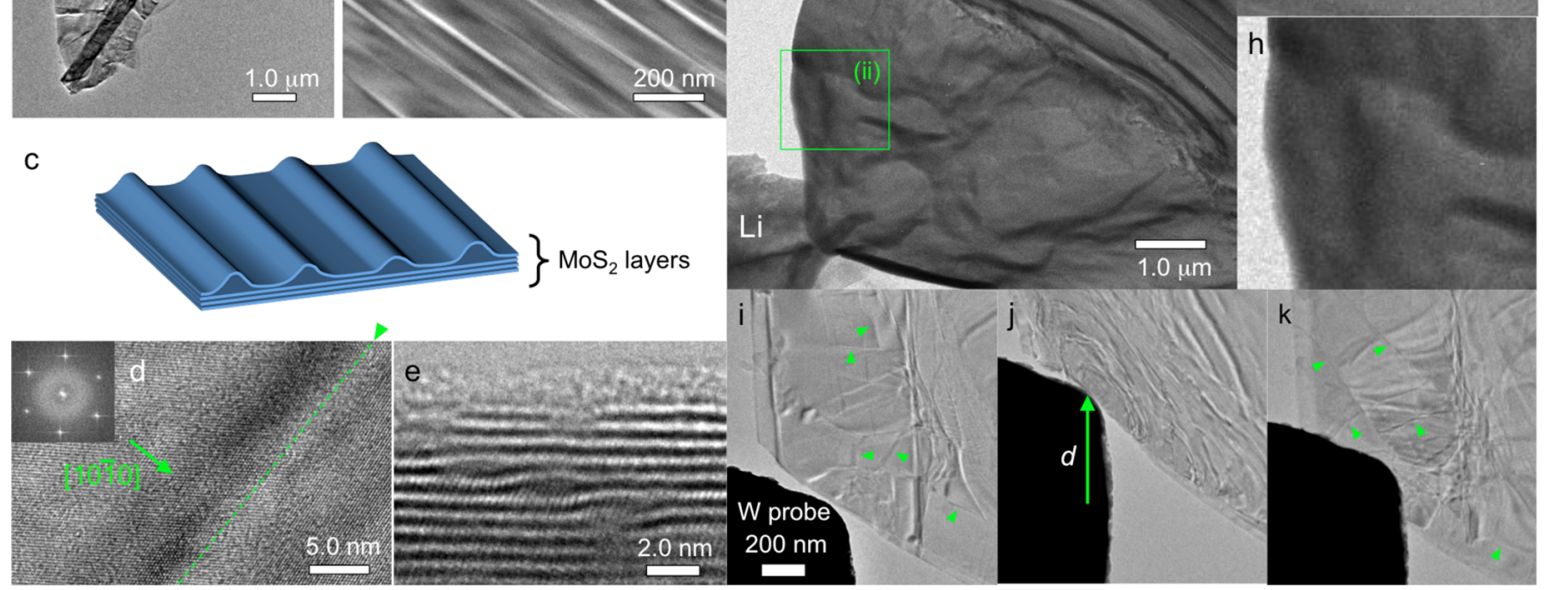

Figure 1. Morphology of ripplocations. (a) A typical TEM image of thin layered $\mathrm{MoS}_{2}$ film. (b) A magnified view of the dashed squared region in (a). The EDP is shown in the inset. (c) Schematic illustration of surface ripplocation in a $\mathrm{MoS}_{2}$ film. (d) A HRTEM image of a ripplocation. (e) A TEM image of cross-section view of a multilayered $\mathrm{MoS}_{2}$ film containing buried ripplocations. (f) Highly mobile ripplocations in a MoS $\mathrm{film}_{2}$ during lithiation (see Supporting Information Movies 1-3). (g) Ripplocations (e.g., the sharp straight line shown in the solid square (i) in f) are

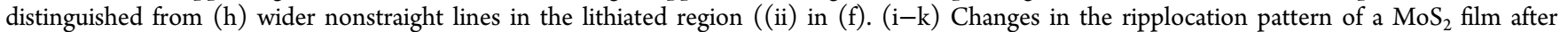
mechanical loading. The initial ripplocations indicated by arrowheads in (i) disappeared after an edge compressive displacement $d=500 \mathrm{~nm}$ along the arrow direction in $(j)$ was applied to the film by a contacting $\mathrm{W}$ probe. When the $\mathrm{W}$ probe was retrieved, new ripplocations were formed as indicated by the arrowheads in (k).

To probe the mobility of ripplocations, we lithiated the $\mathrm{MoS}_{2}$ film using the well-established in situ TEM electrochemomechanical platform ${ }^{12,13}$ as a way to drive ripplocation motion in situ. Lithiation generates a chemical reaction front between unlithiated and lithiated regions. ${ }^{12}$ The shear stress near the propagating electrochemical reaction front drove violent motion of the ripplocation (the sharp line marked by the arrowhead in Figure 1f) (see Supporting Information Movies 1-3 and Figure S3), much like traveling soliton ${ }^{2}$ waves on a water surface. Aside from sharp lines (see Figure 1g, a magnified view of the solid squares (i) in Figure 1f), we also observed tortuous wrinkles with relatively large wavelengths and asymmetric morphologies (see Figure 1h, the magnified views of the solid square (i) in Figure 1f). While sharp ripplocations were observed to be highly mobile, wrinkles on the film at the lithiated region could hardly migrate, once formed. The immobile wrinkles can be analogously found in a floor carpet. We also applied edge compression to the $\mathrm{MoS}_{2}$ film using a tungsten probe to drive ripplocation motion. After the tungsten probe was brought into contact with the $\mathrm{MoS}_{2}$ film at its edge (Figure 1i), a lateral displacement of $\sim 500 \mathrm{~nm}$ was applied parallel to the arrow direction (Figure $1 \mathrm{j}$ ). The loading was then removed by retracting the probe away from the film (Figure 1k). A comparison of the TEM images before and after the loading showed clear differences in the ripplocation patterns, as selectively indicated by the arrowheads in Figure 1i for the disappearing ripplocations and Figure $1 \mathrm{k}$ for the newly emerged ones.

The ease of change in the ripplocation pattern implies that ripplocations can nucleate, migrate, aggregate, and annihilate in response to mechanical, thermal, or chemical perturbations. As ripplocation core does not entail in-plane bond reconstruction, a continuum mechanics based analysis may be adequate to provide the scaling law of the energetics. For a surface ripplocation whose magnitude of Burgers vector $\mathbf{b}$ is much smaller than the arch length $S(b=|\mathbf{b}| \ll S)$, the following geometrical scaling relations hold: $\lambda \sim S, \Delta \sim(b \lambda)^{1 / 2}$, and $\kappa \sim$ $\Delta / \lambda^{2}$, where $\lambda, \Delta$, and $\kappa$ are the projected core width, the height amplitude, and the curvature of the ripplocation, respectively. The total energy $E$ per unit width associated with the ripplocation includes the elastic bending energy $U_{\mathrm{e}} \sim B \lambda \kappa^{2}=$ $B b \lambda^{-2}$ and the interfacial energy $U_{s} \sim \lambda \gamma_{s}$, where $B$ and $\gamma_{s}$ are the bending stiffness and vdW interlayer adhesion energy of the $\mathrm{MoS}_{2}$ film, respectively. Mechanical equilibrium requires $\partial E / \partial \lambda$ $=0$, which leads to a sublinear scaling law, $E \sim b^{1 / 3}$.

The sublinear scaling law above implies that merging of two surface ripplocations is energetically favorable. Such energetics is opposite to Frank's rule for interacting dislocations where same-sign dislocations repel due to the superlinear energy scaling $\left|\mathbf{b}_{1}+\mathbf{b}_{2}\right|^{2}>\left|\mathbf{b}_{1}\right|^{2}+\left|\mathbf{b}_{2}\right|^{2}$. We further confirmed the ripplocation sublinear scaling law by calculating the core configuration and the formation energy of surface ripplocations of different Burgers vectors using ab initio molecular simulations. The surface ripplocations, created by inserting extra $n$ units of $\mathrm{MoS}_{2}$ to the top layer, possess Burgers vectors $\mathbf{b}$ $=n \mathbf{b}_{0}(n=1,2,3, \ldots)$, as schematically described in Figure 2a. The simulation approaches are detailed in Supporting Information. We emphasize that the formation energy (per length) of a ripplocation is well-defined, unlike that of a dislocation in thick, 3D crystals with a long-ranged $(\propto 1 / r)$ elastic field, whose formation energy diverges as the system size increases. ${ }^{14}$ This necessitates the total energy $E_{\text {total }}$ and the core 

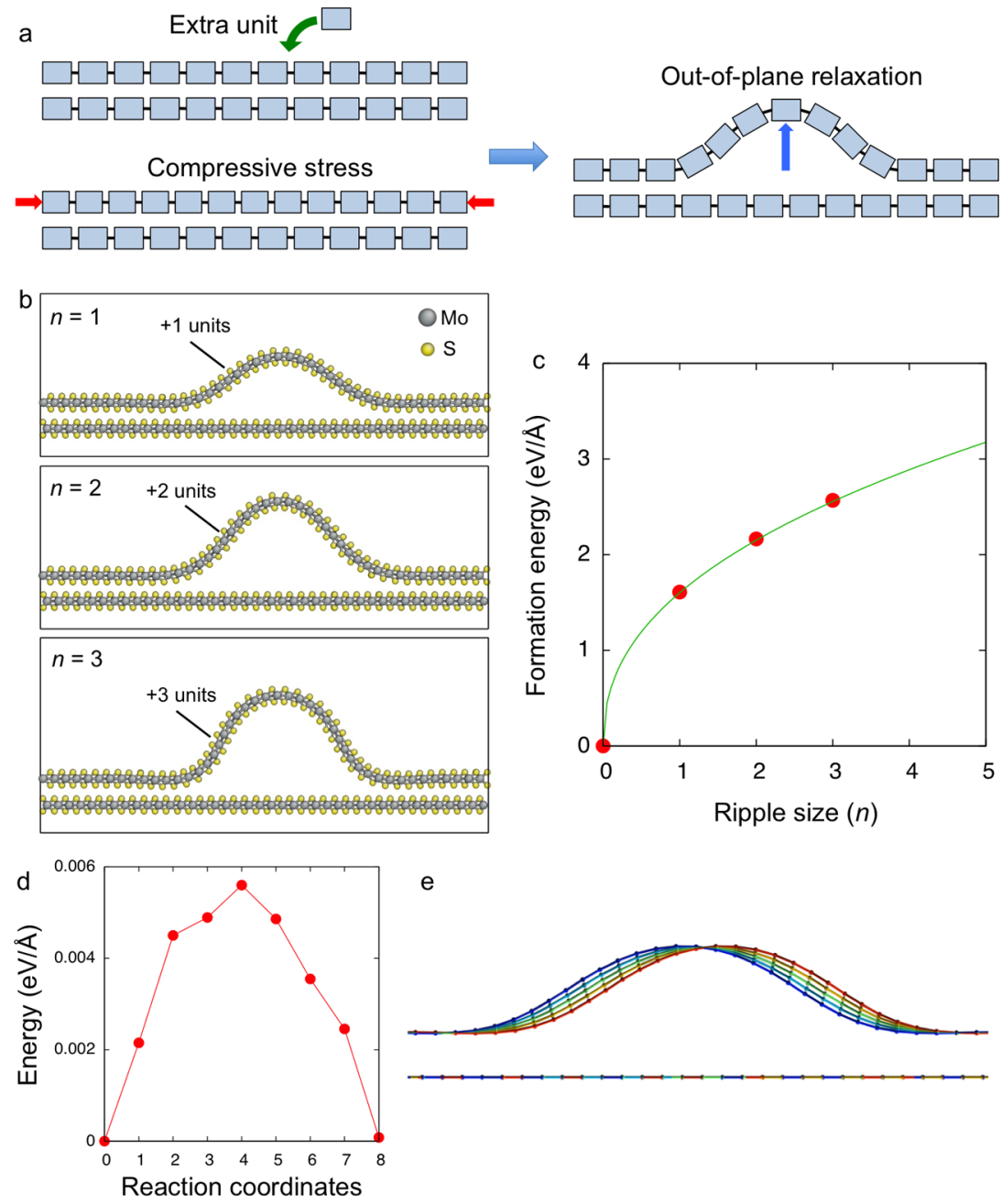

e

f

(i)

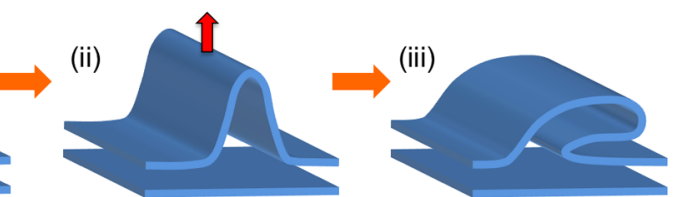

Figure 2. (a) Schematic illustration of the formation of a ripplocation in the simulation by adding an extra unit on the top layer of a MoS $S_{2}$ bilayer. (b) Atomic structures of the ripplocations of different number of extra units $n$ added to the top layer, that is, the Burgers vectors. (c) Formation energy of the ripplocations as a function of $n$. (d) The minimum energy path of the in-plane migration of the unit surface ripplocations calculated by the NEB method. (e) Atomic configurations of the migrating ripplocation along the minimum energy path. Only Mo atoms and Mo-Mo bonds are shown. Blue, cyan, green, yellow, and red colors correspond to the ripplocation structures of the reaction coordinates $0,2,4,6$, and 8 in (d), respectively. (f) Schematic illustration of the aggregation and self-folding processes of the ripplocations.

energy $E_{\text {core }}$ to be separately defined. For a ripplocation, $E_{\text {total }}=$ $E_{\text {core }}$ converges with system size due to its rapidly decaying elastic field. Figure $2 \mathrm{c}$ shows the sublinear scaling of the calculated formation energy with respect to $n$, consistent with the theoretical scaling. Data fitting gives rise to $E \sim n^{0.4}$. The difference between the continuum scaling analysis and the $a b$ initio simulations may stem from the neglect of $\mathrm{vdW}$ interactions at finite interlayer separation $\Delta$ in the ripplocation in the continuum analysis. Our DFT simulations further showed that in the fully relaxed configuration the Mo-Mo bond length across the ripplocations changes by less than $\sim 0.1 \%$ from the flat crystal, indicating a very low in-plane strain. Note that an edge dislocation gliding beneath a free surface in 3D crystals generates a short-ranged $\left(\propto 1 / r^{3}\right)$ elastic strain field and also creates a small ripple on the surface. ${ }^{15}$ What distinguishes ripplocation from the conventional subsurface dislocation is the weak vdW interlayer interactions and the extreme closeness to the vacuum (only one layer), causing interlayer delamination.

The Peierls solution of dislocation core width ${ }^{16}$ and classic Peierls-Nabarro theory ${ }^{17}$ relating dislocation core width to lattice friction do not apply to ripplocation due to different forms of elastic energy for very thin versus thick crystals. To examine the mobility of the ripplocations, we calculated the migration energy landscape of a unit ripplocation by nudged elastic band (NEB) method, ${ }^{18}$ as shown in Figure $2 \mathrm{~d}$. The calculated migration barrier is only $\sim 5.6 \mathrm{meV} / \AA ̊$, which means it takes little external driving force to overcome this barrier and move the ripplocation athermally. ${ }^{19}$ Figure $2 \mathrm{e}$ shows the NEB images along the minimum energy path (MEP). Along the MEP all the atoms constituting the ripplocation were found to move concertedly, resembling the motion of a carpet ruck. 

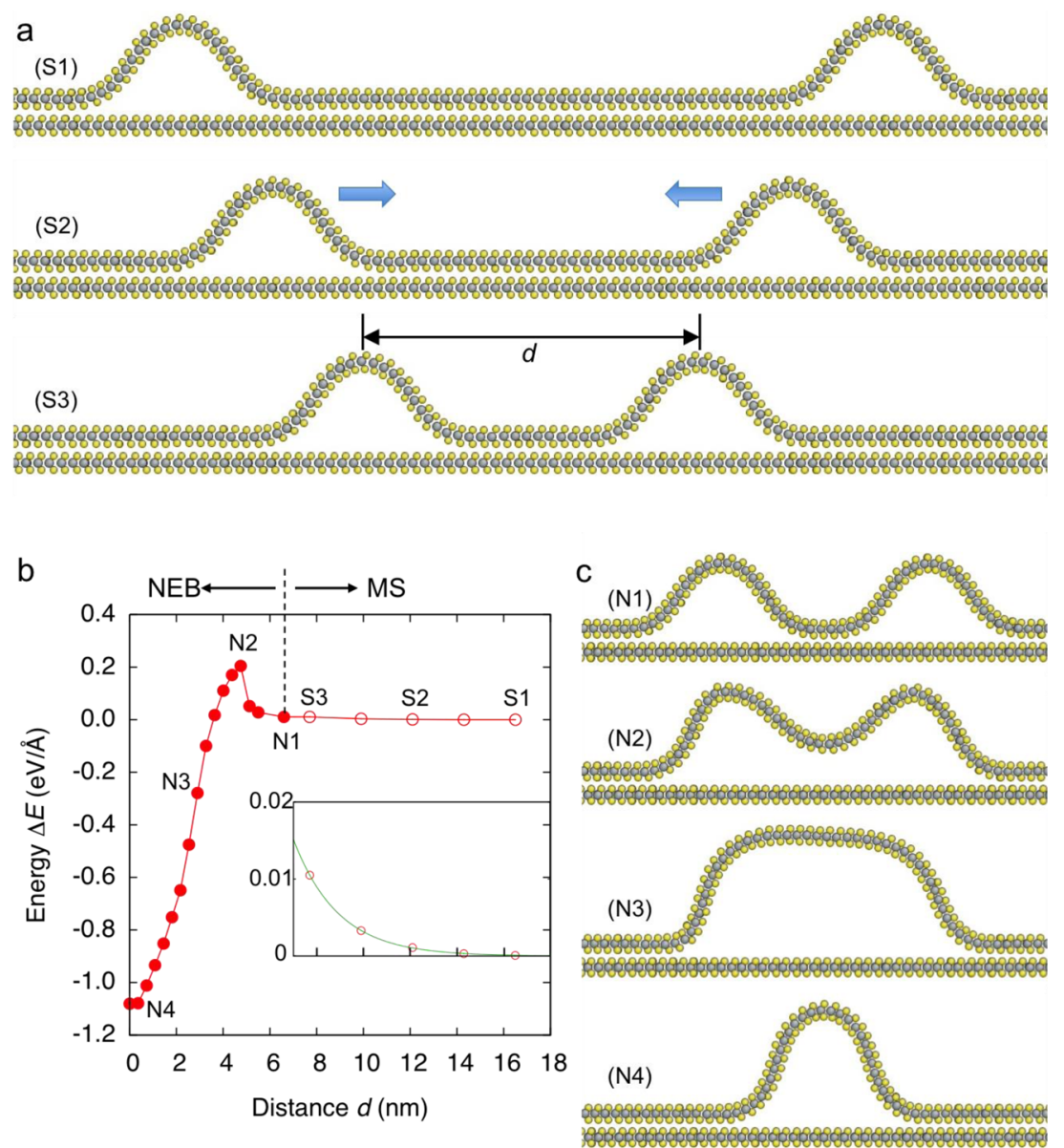

Figure 3. Merging barrier of two ripplocations $(n=3)$ calculated using an empirical force field. (a) Atomic configurations of two ripplocations separated by a distance $d$ calculated by molecular statics (MS) energy minimization. (b) Variations of the system energy $\Delta E$ as a function of $d$. The potential energy at S1 was taken as a reference. Solid and open circles represent the NEB and the MS data, respectively. The inset is the magnified view of the region between S1 and S3. (c) The evolution of the ripplocation structures as they merge, calculated by NEB. Atomic structures S1-S3 in (a) and N1-N4 in (c) correspond to the location labeled on the energy landscape in (b).

Our analysis suggests condensation of ripplocations, which are "quanta" of geometrical incompatibility, into a larger "ripple" that is a more "classical" notion of a bending defect, illustrated in Figure 2f. (In the quantum mechanics analogy, ripplocations are like Bosons.) Beyond a critical magnitude of the Burgers vector, the ripplocation or ripple breaks its leftright symmetry and self-folds into a wrinkle. The wrinkles are kinetically trapped/frustrated because of the multiple interlayer adhesions between self-folded domains, presenting a challenge to eliminate them, similar to a roughened bed sheet on mattress. Such folds were previously introduced in graphene ${ }^{20}$ by transferring graphene onto an etchable substrate, followed by underetching that folds the graphene.

The theoretical results above suggest merging of ripplocations is thermodynamically favorable. We next characterize the merging kinetics of two same-sign ripplocations. Because modeling the interactions of multiple ripplocations is computationally prohibitive for DFT calculations, we resort to empirical force field based atomistic simulation. The empirical potentials $^{21,22}$ were carefully tested and validated against DFT data for elementary material properties (e.g., in-plane and outof-plane moduli, interlayer cohesive energy, and so forth) and able to reproduce the sublinear core-energy scaling law (see Supporting Information). Starting from two well-separated ripplocations in a bilayer $\mathrm{MoS}_{2}$, as shown in S1 of Figure 3a, we decrease the separation distance $d$ between the ripplocations and obtain the atomic configurations in a series of metastable states (e.g., S1, S2, and S3 in Figure 3a) by molecular statics relaxation. The potential energy $\Delta E$ for the metastable states is plotted as a function of $d$, as shown in the inset of Figure 3b. Our result showed there exists a critical separation distance of $d_{\mathrm{c}} \sim 6 \mathrm{~nm}$ (state N1) below which no additional metastable state can be found until the two ripplocations merge completely, indicating that the system encounters a single energy barrier between $0 \leq d<d_{c}$. We then switched to the NEB method to calculate the MEP of the merging process $(\mathrm{N} 1-\mathrm{N} 4)$, where the metastable state of two ripplocations with the shortest possible separation distance (N1) and the fully merge state (N4) were used as the initial and final configurations of the NEB calculation. Our result also shows that the system energy decays exponentially with increasing $d$ for $d>d_{\mathcal{c}}$ indicating that ripplocations are long-distance repulsive. The repulsion, however, is very weak. The potential energy rises sharply as the ripplocations come closer $\left(d<d_{c}\right)$ until it reaches the saddle point of $\Delta E=0.2 \mathrm{eV} / \AA$ at $d_{\text {saddle }}=$ $4.8 \mathrm{~nm}$. Once below the saddle point, the system potential energy decreases rapidly as the two ripplocations merge. The MEP merging processes are shown in Figure 3c. As two ripplocations approached each other, the adherent bilayers between the ripplocations gradually detached, forming a 

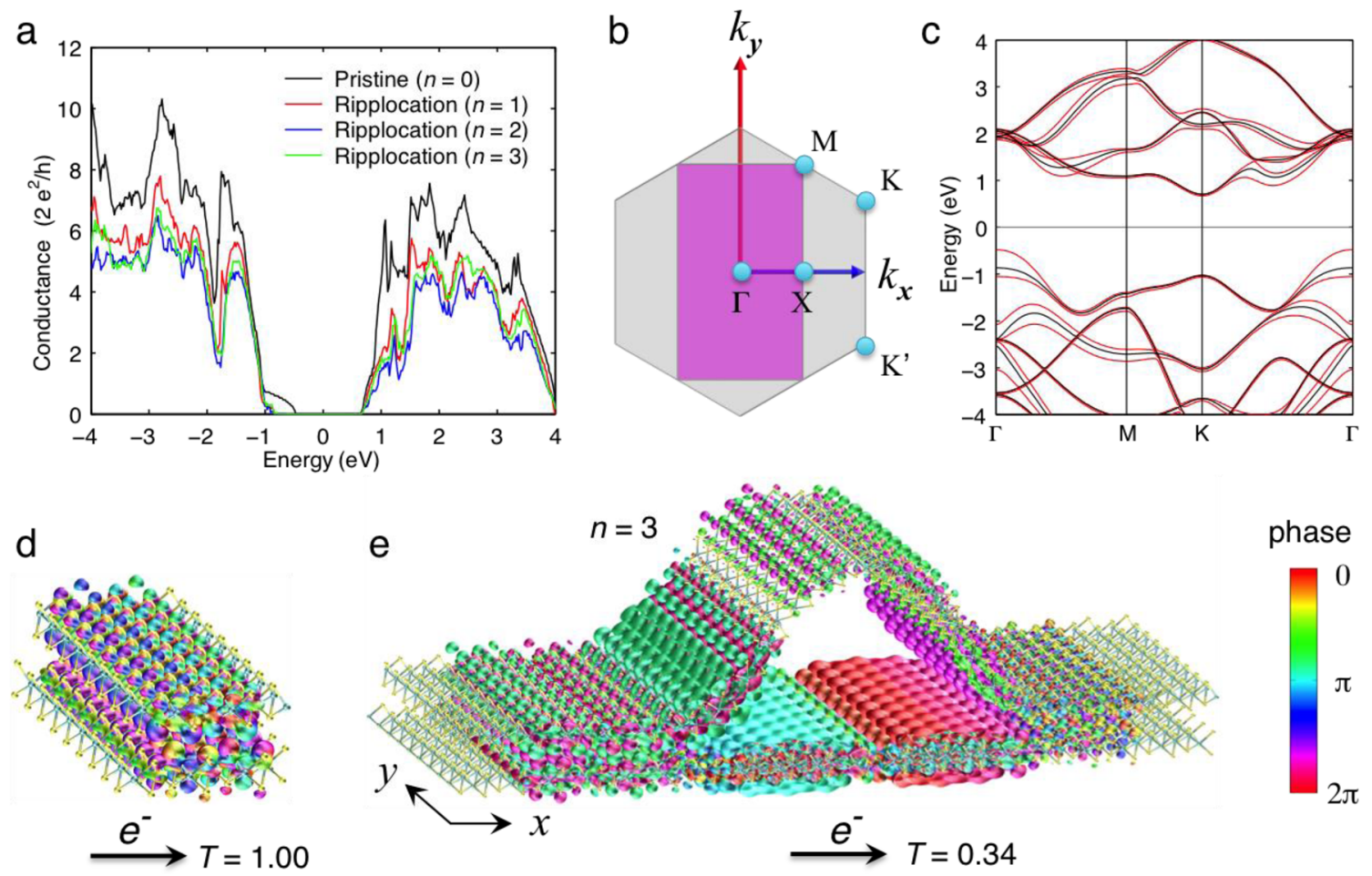

Figure 4. (a) Calculated electrical conductance across a single ripplocation ( $n=1,2$, and 3). (b) Brillouin zones of $\mathrm{MoS}_{2}$ with primitive hexagonal unit cell (gray area) and tetragonal unit cell (purple area). (c) Calculated band structures of the monolayer (black lines) and the bilayer (red lines) $\mathrm{MoS}_{2}$ in hexagonal unit cell. (d,e) Conductance eigenchannels at $(k, E)=(\Gamma,-0.7 \mathrm{eV})$ in bilayer $\mathrm{MoS}_{2}$ without ripple $(n=0)$ and with ripple $(n=$ $3)$, respectively. The color bar depicts the color-encoding scheme of the phase information in the channels.

trapezoidal rack-like saddle configuration. Because the migration barrier is about two orders of magnitude smaller than the merging barrier, multiple ripplocations may also pile up temporarily without merging. This may explain the periodically aligned ripplocations pattern observed in Figure 1b, which may depend on the stress state and temperature. We also note that our calculations did not consider possible chemical adsorbates on surface and/or inside the ripplocation core (e.g., between two layers), which could affect the ripplocation motion.

$\mathrm{MoS}_{2}$ homostructures have bandgaps $\sim 1-2 \mathrm{eV}^{23}$ with potential semiconductor device applications. ${ }^{3}$ Theoretical analyses showed that an inhomogeneous strain distribution can modify the bandgap to create "exciton funnel", ${ }^{24}$ which was recently confirmed experimentally in a $\mathrm{MoS}_{2}$ ripple where an inhomogeneous bending strain makes "excitons drift hundreds of nanometers to lower bandgap regions before recombining" ${ }^{25}$ Controlled unit ripplocations and larger ripples ${ }^{8,9}$ may thus enable $1 \mathrm{D}$ defect engineering. ${ }^{26}$ It is also worth noting that like folded bilayer graphene edges (BLE, "half nanotubes"), ${ }^{27,28}$ ripplocations are very straight, narrow, and crystallographically oriented with highly specific atomic structure. Because only bending and vdW interactions are involved (no in-plane modification of the bonding topology), they have outstanding chemical stability similar to nanotubes, and therefore may be used in devices in a modular fashion. ${ }^{29}$

To investigate this possibility, we calculated electrical conductance along and across ripplocations using firstprinciples nonequilibrium Green's function method ${ }^{30,31}$ within the Landauer-Büttiker formalism. As shown in Figure 4a, the conductance across a single surface ripplocation $(n=1,2$, and 3 ) is significantly reduced compared to the pristine bilayer $\mathrm{MoS}_{2}(n=0)$, and more extra units result in less conductance.
Interestingly, the conductance near the valence band maximum from -1.0 to $0.5 \mathrm{eV}$ is almost completely quenched. To understand this behavior, we calculated the band structure of pristine monolayer and bilayer $\mathrm{MoS}_{2}$ whose Brillouin zone is shown in the gray hexagon of Figure $4 \mathrm{~b}$. The band structure of pristine bilayer $\mathrm{MoS}_{2}$ in Figure 4c clearly shows a significant splitting near the $\Gamma$ point compared to its monolayer. This splitting arises from the interlayer interaction. Because the top valence band near the $\Gamma$ point is higher than the rest valence bands it follows that the quenched conductance arises from these states, as directly verified by the eigenchannel analysis. The conductance eigenchannels at momentum-energy $(k, E)=$ $(\Gamma,-0.7 \mathrm{eV})$ in bilayer $\mathrm{MoS}_{2}$ without ripple $(n=0)$ and with ripple $(n=3)$ are displayed in Figure 4 d,e, respectively. The corresponding phase information is color-encoded in the channels with its colormap shown in the figure. The eigenchannel in the pristine one has $100 \%$ transmission, exhibiting full phase oscillation and constant charge density when electron transports from left to right. However, the eigenchannel in the bilayer $\mathrm{MoS}_{2}$ with the ripplocation $(n=3)$ has only $34 \%$ transmission with the rest of the electron wavepacket backscattered toward the left, resulting in a partial standing-wave whose phase on the left is close to pure real (red) and imaginary (cyan). The transported part on the right side has much reduced magnitude of electron density compared to the left side. A closer look at the bottom layer in Figure 4e reveals a strong quantum-confinement-induced resonance, that is, a large pseudo $p_{x}$ orbital between the disrupted vdW interaction edges close to the two sides of the ripplocation.

Ripplocations exist in different forms: bilayer (only one-layer surface ripples) versus multilayer ripplocations, and surface versus buried ripplocations. The interplay of bending and 
interlayer vdW confinement dictates the resulting morphology, mobility, and interaction of ripplocations. Migrating ripplocations with different line directions may intersect each other, which may locally confine their mobility at the intersections, creating additional folding/frustration. While edge-type surface ripplocations in bilayer homostructures are the primary focus of the present work, we anticipate that our study will stimulate detailed characterizations of the extended forms of this new class of defects, particularly their roles in tailoring mechanical and electronic properties of layered crystals and their interactions with other type of defects such as chemical adsorbates, edges, and so forth. Such fundamental understanding will lay a foundation for controlled defect engineering $^{25,29,32}$ and quality control of layer-by-layer-stacked vdW crystals with tailored functions.

\section{ASSOCIATED CONTENT}

\section{S Supporting Information}

Experimental/simulation procedures, supplementary figures, and movies. This material is available free of charge via the Internet at http://pubs.acs.org.

\section{AUTHOR INFORMATION}

\section{Corresponding Authors}

*(S.Z.) E-mail: suz10@psu.edu.

*(J.L.) E-mail: liju@mit.edu.

\section{Notes}

The authors declare no competing financial interest.

\section{ACKNOWLEDGMENTS}

We acknowledge support from NSF under Awards CBET1240696, DMR-1120901, DMR-1240933, and CMMI0900692. Computational time on the Extreme Science and Engineering Discovery Environment (XSEDE) under the Grants TG-DMR130038 and TG-DMR140003 is gratefully acknowledged. Portions of this work were supported by a Laboratory Directed Research and Development (LDRD) project at Sandia National Laboratories and by Nanostructures for Electrical Energy Storage (NEES), an Energy Frontier Research Center funded by the US Department of Energy (DOE), Office of Science, Office of Basic Energy Sciences (BES) under Award No. DESC0001160.

\section{REFERENCES}

(1) Butz, B.; Dolle, C.; Niekiel, F.; Weber, K.; Waldmann, D.; Weber, H. B.; Meyer, B.; Spiecker, E. Dislocations in bilayer graphene. Nature 2014, 505, 533-537.

(2) Yankowitz, M.; Wang, J. I.-J.; Birdwell, A. G.; Chen, Y.-A.; Watanabe, K.; Taniguchi, T.; Jacquod, P.; San-Jose, P.; Jarillo-Herrero, P.; LeRoy, B. J. Electric field control of soliton motion and stacking in trilayer graphene. Nat. Mater. 2014, 13, 786-789.

(3) Geim, A. K.; Grigorieva, I. V. Van der Waals heterostructures. Nature 2013, 499, 419-425.

(4) Fasolino, A.; Los, J. H.; Katsnelson, M. I. Intrinsic ripples in graphene. Nat. Mater. 2007, 6, 858-861.

(5) Meyer, J. C.; Geim, A. K.; Katsnelson, M. I.; Novoselov, K. S.; Booth, T. J.; Roth, S. The structure of suspended graphene sheets. Nature 2007, 446, 60-63.

(6) Tapasztó, L.; Dumitrică, T.; Kim, S. J.; Nemes-Incze, P.; Hwang, C.; Biró, L. P. Breakdown of continuum mechanics for nanometrewavelength rippling of graphene. Nat. Phys. 2012, 8, 739-742.

(7) Cerda, E.; Mahadevan, L. Geometry and Physics of Wrinkling. Phys. Rev. Lett. 2003, 90, 074302.
(8) Bao, W.; Miao, F.; Chen, Z.; Zhang, H.; Jang, W.; Dames, C.; Lau, C. N. Controlled ripple texturing of suspended graphene and ultrathin graphite membranes. Nat. Nanotechnol. 2009, 4, 562-566.

(9) Schroll, R. D.; Katifori, E.; Davidovitch, B. Elastic Building Blocks for Confined Sheets. Phys. Rev. Lett. 2011, 106, 074301.

(10) Reynwar, B. J.; Illya, G.; Harmandaris, V. A.; Muller, M. M.; Kremer, K.; Deserno, M. Aggregation and vesiculation of membrane proteins by curvature-mediated interactions. Nature 2007, 447, 461464.

(11) Geim, A. K. Graphene: Status and Prospects. Science 2009, 324, $1530-1534$.

(12) Huang, J. Y.; Zhong, L.; Wang, C. M.; Sullivan, J. P.; Xu, W.; Zhang, L. Q.; Mao, S. X.; Hudak, N. S.; Liu, X. H.; Subramanian, A.; Fan, H.; Qi, L.; Kushima, A.; Li, J. In Situ Observation of the Electrochemical Lithiation of a Single $\mathrm{SnO}_{2}$ Nanowire Electrode. Science 2010, 330, 1515-1520.

(13) Kushima, A.; Huang, J. Y.; Li, J. Quantitative Fracture Strength and Plasticity Measurements of Lithiated Silicon Nanowires by In Situ TEM Tensile Experiments. ACS Nano 2012, 6, 9425-9432.

(14) Li, J.; Wang, C.-Z.; Chang, J.-P.; Cai, W.; Bulatov, V.; Ho, K.-M.; Yip, S. Core energy and Peierls stress of a screw dislocation in bcc molybdenum: A periodic-cell tight-binding study. Phys. Rev. B 2004, $70,104113$.

(15) Zandbergen, H.; Pao, C.-W.; Srolovitz, D. Dislocation Injection, Reconstruction, and Atomic Transport on $\{001\}$ Au Terraces. Phys. Rev. Lett. 2007, 98, 036103.

(16) Peierls, R. The size of a dislocation. Proc. Phys. Soc. 1940, 52, 34-37.

(17) Nabarro, F. R. N. Dislocations in a simple cubic lattice. Proc. Phys. Soc. London 1947, 59, 256-272.

(18) Mills, G.; Jónsson, H. Quantum and thermal effects in $\mathrm{H}_{2}$ dissociative adsorption: Evaluation of free energy barriers in multidimensional quantum systems. Phys. Rev. Lett. 1994, 72, 1124.

(19) $\mathrm{Li}$, J. The mechanics and physics of defect nucleation. MRS Bull. 2007, 32, 151-159.

(20) Kim, K.; Lee, Z.; Malone, B. D.; Chan, K. T.; Alemán, B.; Regan, W.; Gannett, W.; Crommie, M. F.; Cohen, M. L.; Zettl, A. Multiply folded graphene. Phys. Rev. B 2011, 83.

(21) Jiang, J.-W.; Park, H. S.; Rabczuk, T. Molecular dynamics simulations of single-layer molybdenum disulphide $\left(\mathrm{MoS}_{2}\right)$ : StillingerWeber parametrization, mechanical properties, and thermal conductivity. J. Appl. Phys. 2013, 114, 064307.

(22) Jiang, J.-W.; Qi, Z.; Park, H. S.; Rabczuk, T. Elastic bending modulus of single-layer molybdenum disulfide $\left(\mathrm{MoS}_{2}\right)$ : finite thickness effect. Nanotechnology 2013, 24, 435705.

(23) Wilson, J. A.; Yoffe, A. D. The transition metal dichalcogenides discussion and interpretation of the observed optical, electrical and structural properties. Adv. Phys. 1969, 18, 193-335.

(24) Feng, J.; Qian, X.; Huang, C. W.; Li, J. Nature Photonics 2012, 6, $866-872$.

(25) Castellanos-Gomez, A.; Roldan, R.; Cappelluti, E.; Buscema, M.; Guinea, F.; van der Zant, H. S. J.; Steele, G. A. Local Strain Engineering in Atomically Thin $\mathrm{MoS}_{2}$. Nano Lett. 2013, 5361-5366.

(26) Maze, J. R.; Stanwix, P. L.; Hodges, J. S.; Hong, S.; Taylor, J. M.; Cappellaro, P.; Jiang, L.; Dutt, M. V. G.; Togan, E.; Zibrov, A. S.; Yacoby, A.; Walsworth, R. L.; Lukin, M. D. Nanoscale magnetic sensing with an individual electronic spin in diamond. Nature 2008, 455, 644-U41.

(27) Huang, J. Y.; Ding, F.; Yakobson, B. I.; Lu, P.; Qi, L.; Li, J. In situ observation of graphene sublimation and multi-layer edge reconstructions. Proc. Natl. Acad. Sci. U.S.A. 2009, 106, 10103-10108.

(28) Feng, J.; Li, W. B.; Qian, X. F.; Qi, J. S.; Qi, L.; Li, J. Patterning of graphene. Nanoscale 2012, 4, 4883-4899.

(29) Qi, J. S.; Huang, J. Y.; Feng, J.; Shi, D. N.; Li, J. The Possibility of Chemically Inert, Graphene-Based All-Carbon Electronic Devices with $0.8 \mathrm{eV}$ Gap. ACS Nano 2011, 5, 3475-3482.

(30) Qian, X.; Li, J.; Yip, S. Calculating phase-coherent quantum transport in nanoelectronics with $a b$ initio quasiatomic orbital basis set. Phys. Rev. B 2010, 82, 195442. 
(31) Brandbyge, M.; Mozos, J.-L.; Ordejón, P.; Taylor, J.; Stokbro, K. Density-functional method for nonequilibrium electron transport. Phys. Rev. B 2002, 65, 165401.

(32) Wu, M.; Qian, X.; Li, J. Tunable Exciton Funnel Using Moiré Superlattice in Twisted van der Waals Bilayer. Nano Lett. 2014, 14, 5350-5357. 